\title{
Time interval to conversion of interferon- $\gamma$ release assay after exposure to tuberculosis
}

\author{
S.W. Lee ${ }^{\star, \#}$, D.K. Oh`, S.H. Lee ${ }^{+}$, H.Y. Kang ${ }^{+}$, C-T. Lee ${ }^{\#}$ and J-J. Yim ${ }^{\S}$
}

ABSTRACT: The proper interval for repeating an interferon- $\gamma$ release assay (IGRA) among tuberculosis contacts with initially negative results is unknown. The interval for IGRA conversion after exposure to patients with active pulmonary tuberculosis in an outbreak setting was evaluated.

In a platoon of $\mathbf{3 2}$ soldiers, four active pulmonary tuberculosis patients, in addition to one index patient, were diagnosed during a contact investigation. For the other 27 contacts, a tuberculin

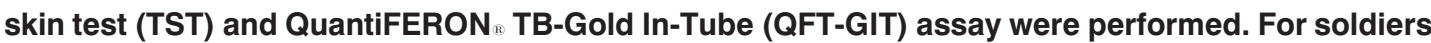
with a negative result on the initial QFT-GIT assay, the test was repeated at 2, 4, 8, 14, 18 and 30 weeks until positive conversion occurred. When conversion was identified, the subject was treated for latent tuberculosis infection.

Initially, 17 (63.0\%) soldiers gave positive QFT-GIT results, whereas $21(77.8 \%)$ showed positive TST results. Among 10 participants with initially negative QFT-GIT results, three showed conversion at 2 weeks, three at 4 weeks and three at 14 weeks. Conversion did not occur during the 30-week observation period in one contact.

Based on the tuberculosis exposure time-points among the contacts, IGRA conversion generally occurred 4-7 weeks after exposure, although it could occur as late as 14-22 weeks after exposure.

KEYWORDS: Contacts, infection control, interferon- $\gamma$ assay, serial testing, tuberculosis

D espite global efforts, the total number of new tuberculosis (TB) cases continues to rise, with 9.27 million new cases and 1.32 million deaths reported in 2007 [1]. For effective and efficient treatment of active TB, and for disease control in developing countries, the rapid diagnosis and treatment of patients with sputum smear-positive $\mathrm{TB}$ are critical. In countries with low or intermediate rates of $\mathrm{TB}$, the treatment of latent TB infection (LTBI) to prevent progression to active disease has been an essential component of public health efforts to eliminate the disease $[2,3]$.

Despite its well-known limitations, the tuberculin skin test (TST) has been the only method of diagnosing LTBI for over a century. However, two interferon (IFN) $-\gamma$ release assays (IGRAs) that overcome several TST limitations were recently introduced into routine clinical practice. The IGRAs appear to be unaffected by previous bacille Calmette-Guérin (BCG) vaccination. They do not require intradermal injection or a second visit. In addition, IGRAs provide objective results.

Compared with the TST, two commercially available tests, the ELISA-based QuantiFERON ${ }_{\circledast}$ TBGold assay (Cellestis, Carnegie, Australia) and the enzyme-linked immunospot-based T-SPOT.TB test (Oxford Immunotec, Abingdon, UK), produce more specific results [4, 5]. The UK National Institute for Health and Clinical Excellence (NICE) guidelines (covering England) recommend the use of IGRAs in individuals at risk of LTBI who have tested positive with TSTs [6]. The US Centers for Disease Control and Prevention (CDC) guidelines recommend initial use of IGRAs in all groups as a direct replacement for the TST [7].

Nevertheless, the interval for positive conversion of the IGRA following exposure to a patient with active TB is unclear, whereas conversion of the TST is known to occur within 2-12 weeks [8-10]. NICE guidelines recommend repetition of an IGRA test after 6 weeks for contacts with negative TST results, although there is no concrete evidence for this recommendation [6]. Similarly, CDC guidelines recommend that negative IGRA results be confirmed with a repeat test performed 8-10 weeks after the end of exposure, while admitting that the best interval for requesting a retest is unknown [7].

In the present study, the time interval for positive IGRA conversion after a TB outbreak was evaluated in a Korean military platoon, which is a closed communal setting.
AFFILIATIONS

*Dept of Internal Medicine

'Dept of Radiology, Armed Forces Capital Hospital

\#Dept of Internal Medicine, Seoul

National University Bundang

Hospital, Seongnam,

${ }^{+}$Molecular Biology Section, Korean Institute of Tuberculosis, and

${ }^{\S}$ Division of Pulmonary and Critical

Care Medicine, Dept of Internal

Medicine and Lung Institute, Seoul

National University College of

Medicine, Seoul, Republic of Korea.

CORRESPONDENCE

J-J. Yim

Division of Pulmonary and Critical

Care Medicine, Dept of Internal

Medicine and Lung Institute

Seoul National University College of

Medicine

101 Daehangno

Jongno-gu

Seoul

110-744

Republic of Korea

E-mail: yimjj@snu.ac.kr

Received:

Jun 102010

Accepted after revision:

Nov 072010

First published online:

Dec 092010 


\section{MATERIALS AND METHODS}

\section{Status of TB control in South Korea}

In 2007, the incidence and prevalence of TB in South Korea were 90 and 126 per 100,000 population, respectively. In addition, annual TB mortality was 4,887 [1], and the rate of BCG coverage was $\sim 95 \%$ [11]. The prevalence of positive TST results among young South Koreans without contact with TB patients was reported to be $28 \%$ in 2006 [12].

\section{Index patient and identification of the TB outbreak}

The first patient diagnosed with pulmonary TB was a 20-yr-old male soldier in a platoon of two officers and 32 soldiers. This soldier had no underlying disease and was HIV-negative. The soldier developed a productive cough at the beginning of January 2009 and showed haemoptysis in March 2009. Subsequently, the soldier was diagnosed with sputum smearpositive pulmonary TB in April 2009, and was evacuated from the platoon for isolation. The soldier was treated for active pulmonary TB and, 5 weeks later, culture confirmed Mycobacterium tuberculosis infection. Within 7 days of this patient's diagnosis, simple chest radiography (CXR) was performed for all of the officers and soldiers in the same platoon. Acid-fast staining and mycobacterial sputum culture were also conducted for any contacts who could expectorate sputum.

\section{Study protocol}

On May 2009, the investigation of the present study was initiated for all of the soldiers in the same platoon. Although the soldiers in the platoon lived in two adjacent rooms, they shared a bathroom and dining room, and lived their daily lives together. Therefore, all of them were invited to participate in the present study. However, two officers were excluded from the study because they did not have such close contact as the soldiers themselves. After giving written consent, each participant was asked to complete a questionnaire regarding demographics, previous history of $\mathrm{TB}$, smoking status and other factors. Additionally, chest low-dose computed tomography (LDCT), a TST and a QuantiFERON ${ }_{\star}$ TB-Gold In-Tube (QFT-GIT) assay (Cellestis), as well as acid-fast bacillus (AFB) smears and mycobacterial culture of sputum where possible, were performed for all close contacts (fig. 1). LDCT was included to detect any active lesions that were not identified by CXR $[13,14]$. The present study was reviewed and approved by the institutional review board of the Armed Forces Medical Command of the Republic of Korea.

\section{Tuberculin skin test}

After QFT-GIT blood sampling, the two-step TST was performed by trained personnel following standard procedures [9], with $0.1 \mathrm{~mL}$ (2 tuberculin units) purified protein derivative (RT23; Statens Serum Institute, Copenhagen, Denmark). A positive test was defined as an induration of $\geqslant 10 \mathrm{~mm}$ [9].

\section{Interferon- $\gamma$ release assay}

The QFT-GIT assay was performed according to the manufacturer's instructions. The plasma concentration of IFN- $\gamma$ was measured by ELISA, and the technician had no information about the subjects. Test results were interpreted as negative, indeterminate or positive (cut-off $0.35 \mathrm{IU} \cdot \mathrm{mL}^{-1}$ ) using the manufacturer's software. QFT-GIT conversion was defined as a change from a negative $\left(<0.35 \mathrm{IU} \cdot \mathrm{mL}^{-1}\right)$ to a positive $\left(\geqslant 0.35 \mathrm{IU} \cdot \mathrm{mL}^{-1}\right)$ result [15].

\section{Chest LDCT}

LDCT was performed using a 64-channel multidetector (Brilliance 64; Philips, Amsterdam, the Netherlands) and a thickness of $5 \mathrm{~mm}$. A board-certified radiologist (certification performed by Ministry of Health and Welfare, Seoul, Republic of Korea), who was blinded to the participants' clinical information, interpreted the results.

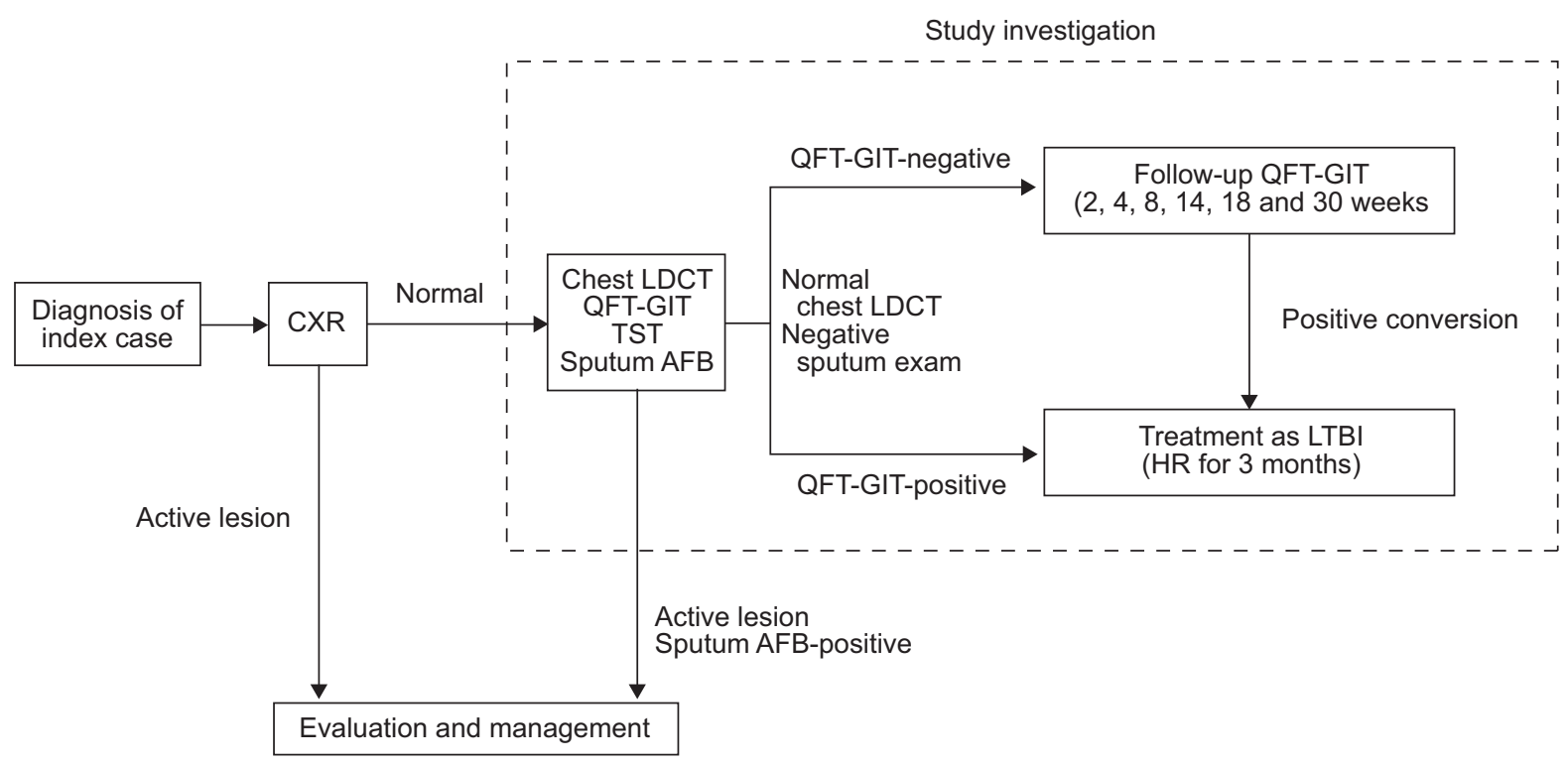

FIGURE 1. Schematic of the study protocol. CXR: chest radiography; LDCT: low-dose computed tomography; QFT-GIT: QuantiFERON w TB-Gold In-Tube; TST: tuberculin skin test; AFB: acid-fast bacillus; exam: examination; LTBI: latent tuberculosis infection; HR: isoniazid/rifampicin. 
TABLE 1 Demographic and clinical characteristics of five patients with pulmonary tuberculosis

\begin{tabular}{|c|c|c|c|c|c|c|c|}
\hline $\begin{array}{l}\text { Patient } \\
\text { No. }\end{array}$ & $\begin{array}{r}\text { Age } \\
\text { yrs }\end{array}$ & $\begin{array}{c}\text { Symptom } \\
\text { duration }^{\#} \\
\text { weeks }\end{array}$ & $\begin{array}{c}\text { Time from } \\
\text { diagnosis } \\
\text { weeks }\end{array}$ & $\begin{array}{c}\text { AFB sputum } \\
\text { smear }\end{array}$ & $\begin{array}{l}\text { Mycobacterial } \\
\text { sputum culture }\end{array}$ & RFLP pattern & Radiographic findings \\
\hline 1 & 21 & 12 & 4 & Positive & M. tuberculosis & Index case & Cavitary consolidation in RUL \\
\hline 2 & 21 & 4 & 3 & Negative & M. tuberculosis & Same as index & Consolidation in RML \\
\hline 3 & 21 & 5 & 3 & Negative & M. tuberculosis & Different from index & Bronchiolitis in LLL \\
\hline 5 & 22 & No symptoms & 0 & Negative & Negative & NA & Bronchiolitis in RUL \\
\hline
\end{tabular}

Patients 3 and 5 were diagnosed based on abnormal low-dose computed tomography chest lesions. AFB: acid-fast bacillus; RFLP: restriction fragment length polymorphism; M. tuberculosis: Mycobacterium tuberculosis; RUL: right upper lobe; RML: right middle lobe; LLL: left lower lobe; NA: not applicable. \#: duration of respiratory symptoms before isolation; ${ }^{\bullet}$ : to investigation

\section{AFB smears and mycobacterial sputum culture}

For participants who could expectorate sputum, three AFB smears and mycobacterial culture of the sputum were performed. Sputum was decontaminated with $4 \% \mathrm{NaOH}$, homogenised and concentrated by centrifugation for $20 \mathrm{~min}$ at $3,000 \times g$. The processed sediment was stained using the ZiehlNeelsen method [16]. Sputum was also cultured in 3\% Ogawa medium and observed for growth every week for 8 weeks.

\section{Diagnosis and treatment of active TB and LTBI}

Active TB

Active TB was diagnosed when specimens showed positive AFB staining, M. tuberculosis was cultured or patient data met the definition of a clinical case of TB as defined by the World Health Organization [17]. Since the isolated M. tuberculosis showed susceptibility to all anti-TB drugs, treatment with isoniazid, rifampicin, pyrazinamide and ethambutol was initiated and continued for 6 months for participants diagnosed with active TB.

\section{LTBI}

Participants with positive QFT-GIT results, but without active TB lesions on CXR and LDCT, were diagnosed with LTBI, and daily isoniazid and rifampicin were recommended for 3 months [18].

\section{Follow-up evaluation}

Participants with negative QFT-GIT results on initial screening were tested again at 2, 4, 8, 14, 18 and 30 weeks after the outbreak investigation had begun. When a positive QFT-GIT conversion occurred, the follow-up tests were stopped, and the participant was treated daily with isoniazid and rifampicin for 3 months (fig. 1).

\section{DNA fingerprinting of isolated $\mathrm{M}$. tuberculosis}

In order to confirm an outbreak of $M$. tuberculosis at the molecular level, DNA fingerprinting was performed using PvuII (Proteus vulgaris II) restriction, as described previously [19].

\section{Statistical analysis}

Agreement between the TST results and whole-blood IFN- $\gamma$ levels was measured using the $\kappa$ statistic. Statistical significance was defined by a two-tailed $\mathrm{p}$-value of $\leqslant 0.05$. All statistical analyses were conducted using PASW 17.0 (SPSS, Inc., Chicago, IL, USA).

\section{RESULTS}

\section{Patients identified with active pulmonary TB}

During the investigation, two more soldiers with productive cough were diagnosed with active pulmonary $\mathrm{TB}$ based on symptoms and CXRs, despite giving negative AFB sputum smear results; after a few weeks, M. tuberculosis was isolated from one of the two soldiers. In addition, two more soldiers with normal CXRs were diagnosed and treated for active TB based on abnormal LDCT lesions; although their AFB smears were negative, $M$. tuberculosis was isolated from the sputum of one of the soldiers several weeks later. In total, five soldiers in the present platoon, including the index case, were diagnosed with active pulmonary TB. Three of the soldiers had cultureconfirmed pulmonary TB. All five patients were isolated from the platoon as soon as active TB was suspected. Their symptoms and radiographic findings improved with anti-TB treatment. M. tuberculosis cultured from two patients (index patient and one subsequent patient who had lesions on CXR) showed the same restriction fragment length polymorphism pattern; however, the patient diagnosed as having active TB based on LDCT showed a different pattern of $M$. tuberculosis (table 1).

\section{Demographic characteristics of contacts without active TB}

All 27 contacts in the platoon with normal LDCT results completed the study protocol. Their median age was $21 \mathrm{yrs}$ (range 20-24 yrs), and all were male. A total of $21(77.8 \%)$ contacts had BCG scars, and $19(70.4 \%)$ were current smokers. All contacts were negative for HIV, and none had a previous history of TB. A total of 16 contacts were residing with the platoon when the index case started to cough; seven contacts were deployed to the platoon before the diagnosis and isolation of the index case, and four contacts were deployed to the platoon after isolation of the index case, but were exposed to the four other patients with active TB.

\section{Results of the initial investigation for contacts without active TB}

Among the 27 contacts, $17(63.0 \%)$ gave a positive QFT-GIT result and none had an indeterminate result, whereas $21(77.8 \%)$ showed a positive response on the TST. Of the 


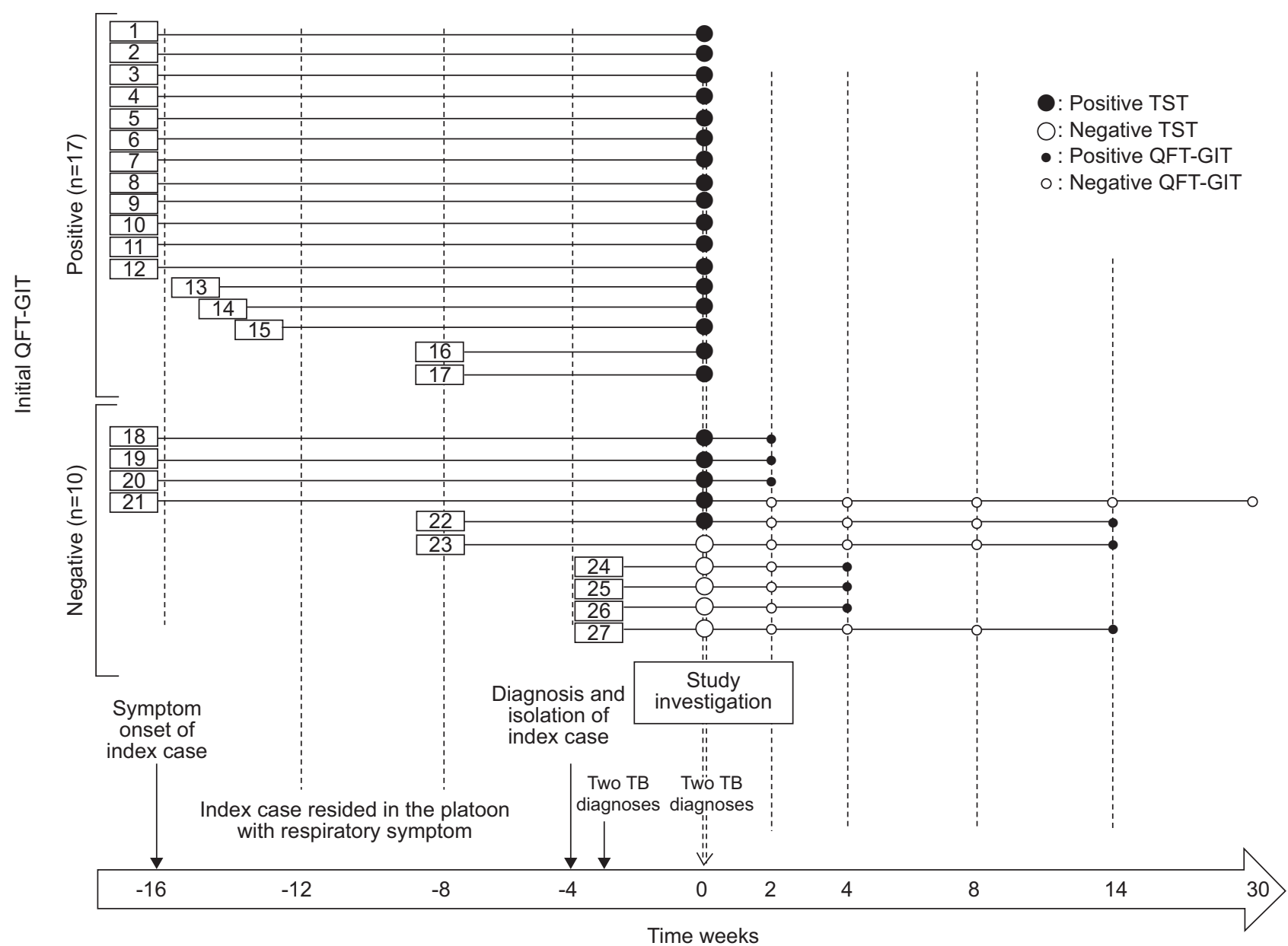

FIGURE 2. Interval between tuberculosis (TB) exposure and QuantiFERON $N^{*}$ TB-Gold In-Tube (QFT-GIT; Cellestis, Carnegie, Australia) conversion among 27 contacts without active TB. Each numbered square represents one participant and is placed at the point at which the participant started to reside with the platoon. TST: tuberculin skin test.

contacts, $16(59.3 \%)$ gave positive results on both the QFT-GIT assay and TST, and five (18.5\%) gave negative results on both tests. The overall agreement between the TST and QFT-GIT results was moderate $(\kappa=0.56 ; p=0.001)$ [20].

\section{Follow-up IGRAs among contacts with initially negative results}

A total of 10 contacts gave negative QFT-GIT results during the initial study investigation. Among them, three participants (all with a positive TST result in the first step) showed conversion at 2 weeks, with IFN- $\gamma$ levels of 0.51 (subject 18), >10.0 (subject 19) and $1.47 \mathrm{IU} \cdot \mathrm{mL}^{-1}$ (subject 20). Another three (all with a negative TST result) showed conversion at 4 weeks, with IFN- $\gamma$ levels of 0.58 (subject 24), 0.90 (subject 25) and $0.96 \mathrm{IU} \cdot \mathrm{mL}^{-1}$ (subject 26). Three other participants (one with a positive TST result in the first step and two with a negative TST result) showed conversion at 14 weeks; the IFN- $\gamma$ levels were 1.09 (subject 22), 1.92 (subject 23) and $2.74 \mathrm{IU} \cdot \mathrm{mL}^{-1}$ (subject 27). No conversion occurred in one contact (subject 21) during the 30-week observation period (fig. 2).

\section{Subsequent development of active TB among participants}

None of the 26 contacts were diagnosed with active TB during the 10-month follow-up period.

\section{DISCUSSION}

Current guidelines recommend repeating the IGRA at 6-10 weeks after TB exposure among contacts with an initially negative IGRA result [6, 7]. However, this recommendation has not emerged from observed data, but from speculation. Using the results of the present study, the interval between exposure and IGRA conversion could be estimated.

The results of the serial QFT-GIT assay in subjects 24-26 showed the shortest interval to conversion. Given that these three subjects had been deployed to the platoon 3 weeks before the investigation began, and considering that conversion occurred at 4 weeks after the investigation began, the interval from exposure to conversion must have been 4-7 weeks for these subjects. The longest interval to conversion was also estimated using the data from subjects 22 and 23, who could have contacted persons with active TB as long as 2 months before the investigation and showed conversion 14 weeks after the investigation began. Based on the present results, conversion is possible as early as 4-7 weeks and as late as 14-22 weeks after exposure to $\mathrm{TB}$, although contacts were not checked for $>6$ months.

The present study was performed to estimate the interval between exposure and IGRA conversion under ideal 
conditions. First, the participants in the present study had scant possibility of further exposure to other patients with active TB. In a platoon of 32 soldiers, five with active TB were isolated as soon as active TB was suspected. Although two participants with initially negative IGRA results had spent a few days outside the barracks before IGRA conversion, they denied contact with TB patients while they were outside. None of the other participants left their platoon for holiday before IGRA conversion. Furthermore, the possibility of missing a patient with active TB was minimised by performing LDCT for all contacts, as LDCT has been shown to be superior to a simple CXR for diagnosing active TB $[13,14]$. Indeed, two patients in the present investigation were diagnosed with active pulmonary TB based on LDCT. Furthermore, contact between the participants and soldiers from other units was very unlikely as the platoon had performed their duties independently in an isolated barracks. We believe that, in this setting, the observed positive conversions resulted from exposure to the index patient or subsequent patients with $\mathrm{TB}$, rather than from other sources. Secondly, none of the contacts with a negative QFT-GIT result were released from military service. This condition provided an ideal opportunity to observe the whole set of contacts and minimised selection bias. The high rate of positive TST (21 out of 27) and QFT-GIT results (17 out of 27) among the contacts at the first screening raised the possibility of widespread pre-existing LTBI among the soldiers; however, a large proportion of the LTBI among the participants was thought to be the result of the intense exposure to the TB case, given that the prevalence of positive TST results among young South Koreans without contact with TB patients is only $28 \%$ in South Korea [12].

Theoretically, the TST could affect the results of subsequent IGRA tests, because both 6-kDa early secretory antigenic target and culture filtrate protein 10, which are used in the IGRA, are included in tuberculin. However, the data regarding a boosting effect of the TST on IGRA assays are inconsistent across studies, and some previous studies confute a definite boosting of IGRA response by the TST [21-23].

Owing to within-subject variability in the IFN- $\gamma$ responses [24], a higher threshold $\left(\geqslant 0.70 \mathrm{IU} \cdot \mathrm{mL}^{-1}\right)$ has been suggested to define conversion for the QFT-GIT assay [25]. Without a higher threshold, minor fluctuations around the current IFN- $\gamma$ cut-off level of $0.35 \mathrm{IU} \cdot \mathrm{mL}^{-1}$ could be mistakenly regarded as conversions. In the present study, the participants showing conversion had IFN- $\gamma$ levels of $>0.70 \mathrm{IU} \cdot \mathrm{mL}^{-1}$, with the exception of two participants (IFN- $\gamma$ level of $0.51 \mathrm{IU} \cdot \mathrm{mL}^{-1}$ in subject 6 and $0.58 \mathrm{IU} \cdot \mathrm{mL}^{-1}$ in subject 27). Considering previous studies, the high IFN- $\gamma$ levels of participants in the present study can be regarded as real conversions, rather than boosting from the TST or within-subject variability in the IFN- $\gamma$ response.

Given the TB exposure time-points of contacts, IGRA conversion generally occurred 4-7 weeks after exposure, and occurred as late as 14-22 weeks after exposure in a smaller number of cases. No conversion was observed 22-30 weeks after the outbreak investigation. Thus, for close contacts with an initially negative IGRA result, the appropriate time over which to repeat the IGRA may be longer than the currently recommended 6-10 weeks.

\section{SUPPORT STATEMENT}

This study was supported by the Korean Military Medical Research Project funded by the Ministry of National Defense of the Republic of Korea (Seoul, Republic of Korea).

\section{STATEMENT OF INTEREST}

None declared.

\section{REFERENCES}

1 World Health Organization. Global Tuberculosis Control: Epidemiology, Strategy, Financing: WHO Report 2009. Report No. WHO/ HTM/TB/2009.411. Geneva, World Health Organization, 2009.

2 Broekmans JF, Migliori GB, Rieder HL, et al. European framework for tuberculosis control and elimination in countries with a low incidence. Eur Respir J 2002; 19: 765-775.

3 Jasmer RM, Nahid P, Hopewell PC. Clinical practice. Latent tuberculosis infection. N Engl J Med 2002; 347: 1860-1866.

4 Kang YA, Lee HW, Yoon HI, et al. Discrepancy between the tuberculin skin test and the whole-blood interferon $\gamma$ assay for the diagnosis of latent tuberculosis infection in an intermediate tuberculosis-burden country. JAMA 2005; 293: 2756-2761.

5 Menzies D, Pai M, Comstock G. Meta-analysis: new tests for the diagnosis of latent tuberculosis infection: areas of uncertainty and recommendations for research. Ann Intern Med 2007; 146: 340-354.

6 National Institute for Health and Clinical Excellence. Tuberculosis: Clinical Diagnosis and Management of Tuberculosis, and Measures for its Prevention and Control. Clinical Guideline 33. London, National Institute for Health and Clinical Excellence, 2006.

7 Mazurek M, Jereb J, Vernon A, et al. Updated guidelines for using interferon gamma release assays to detect Mycobacterium tuberculosis infection - United States, 2010. MMWR Recomm Rep 2010; 59: 1-25.

8 Wasz-Hockert O. On the period of incubation in tuberculosis. Ann Med Intern Fenn 1947; 96: 764-772.

9 Targeted tuberculin testing and treatment of latent tuberculosis infection. Am J Respir Crit Care Med 2000; 161: S221-S247.

10 Huebner RE, Schein MF, Bass JB Jr. The tuberculin skin test. Clin Infect Dis 1993; 17: 968-975.

11 Kim HJ, Oh SY, Lee JB, et al. Tuberculin survey to estimate the prevalence of tuberculosis infection of the elementary schoolchildren under high BCG vaccination coverage. Tuberc Respir Dis 2008; 65: 269-276.

12 Choi CM, Kang CI, Kim DH, et al. The role of TST in the diagnosis of latent tuberculosis infection among military personnel in South Korea. Int J Tuberc Lung Dis 2006; 10: 1342-1346.

13 Lee SW, Jang YS, Park CM, et al. The role of chest CT scanning in TB outbreak investigation. Chest 2010; 137: 1057-1064.

14 Lew WJ, Jung YJ, Song JW, et al. Combined use of QuantiFERON ${ }_{\mathbb{R}-}$ TB Gold assay and chest computed tomography in a tuberculosis outbreak. Int J Tuberc Lung Dis 2009; 13: 633-639.

15 Cellestis, Inc. QuantiFERON Insert. Valencia, CA, Cellestis, Inc., 2006. www.cellestis.com/IRM/ Content/pdf/QuantiFeron\%20US\%20VerH_Jul2010.pdf Date last updated: July 2010. Date last accessed: October 7, 2010.

16 World Health Organization. Laboratory Services in Tuberculosis Control. Geneva, World Health Organization, 1998.

17 World Health Organization. Treatment of Tuberculosis: Guidelines for National Programmes. 3rd Edn. Geneva, World Health Organization, 2003.

18 Joint Tuberculosis Committee of the British Thoracic Society. Control and prevention of tuberculosis in the United Kingdom: code of practice 2000. Thorax 2000; 55: 887-901.

19 van Embden JD, Cave MD, Crawford JT, et al. Strain identification of Mycobacterium tuberculosis by DNA fingerprinting: recommendations for a standardized methodology. J Clin Microbiol 1993; 31: 406-409. 
20 Landis JR, Koch GG. The measurement of observer agreement for categorical data. Biometrics 1977; 33: 159-174.

21 Naseer A, Naqvi S, Kampmann B. Evidence for boosting Mycobacterium tuberculosis-specific IFN- $\gamma$ responses at 6 weeks following tuberculin skin testing. Eur Respir J 2007; 29: 1282-1283.

22 Igari $\mathrm{H}$, Watanabe A, Sato T. Booster phenomenon of QuantiFERON-TB Gold after prior intradermal PPD injection. Int J Tuberc Lung Dis 2007; 11: 788-791.
23 Vilaplana C, Ruiz-Manzano J, Gil O, et al. The tuberculin skin test increases the responses measured by $\mathrm{T}$ cell interferon- $\gamma$ release assays. Scand J Immunol 2008; 67: 610-617.

24 van Zyl-Smit RN, Pai M, Peprah K, et al. Within-subject variability and boosting of T-cell interferon- $\gamma$ responses after tuberculin skin testing. Am J Respir Crit Care Med 2009; 180: 49-58.

25 Pai M, Joshi R, Dogra S, et al. Serial testing of health care workers for tuberculosis using interferon- $\gamma$ assay. Am J Respir Crit Care Med 2006; 174: 349-355. 